\title{
LINEAR OPERATORS PRESERVING THE NUMERICAL RADIUS OF MATRICES
}

\author{
CHI-KWONG LI
}

\begin{abstract}
In this note we characterize all the linear operators on the linear space of $n \times n$ complex matrices which preserve the numerical radius and all the linear operators on the real linear space of $n \times n$ Hermitian matrices which preserve the numerical radius. From our results, we easily deduce V. J. Pellegrini's characterization of all linear operators that preserve the numerical ranges of matrices and the result of Marcus and Moyls concerning the linear operators that preserve the eigenvalues of Hermitian matrices.
\end{abstract}

Results. Let $\mathbf{C}_{n \times n}$ be the complex linear space of all $n \times n$ complex matrices and denote by $\mathcal{H}_{n}$ the real linear space of all $n \times n$ Hermitian matrices. For a matrix $A$ in $\mathbf{C}_{n \times n}$ define the numerical range of $A$ to be the set

$$
W(A)=\left\{x A x^{*}: x \in \mathbf{C}^{n}, x x^{*}=1\right\}
$$

in C. Associated with the numerical range of $A$ is the quantity

$$
r(A)=\max \{|z|: z \in W(A)\},
$$

which is known as the numerical radius of $A$.

The purpose of this note is to prove the following results.

THEOREM 1. A linear operator $T: \mathbf{C}_{n \times n} \rightarrow \mathbf{C}_{n \times n}$ satisfies

$$
r(T(A))=r(A) \text { for all } A \text { in } \mathbf{C}_{n \times n}
$$

if and only if there exist a unitary matrix $U$ in $\mathbf{C}_{n \times n}$ and $\xi$ in $\mathbf{C}$ with $|\xi|=1$ such that

$$
T(A)=\xi U A U^{*} \text { for all } A \text { in } \mathbf{C}_{n \times n},
$$

or

$$
T(A)=\xi U A^{T} U^{*} \quad \text { for all } A \text { in } \mathbf{C}_{n \times n} .
$$

THEOREM 2. A linear operator $T: \mathscr{H}_{n} \rightarrow \mathfrak{H}_{n}$ satisfies

$$
r(T(A))=r(A) \text { for all } A \text { in } \not{H}_{n}
$$

if and only if there exist a unitary matrix $U$ in $\mathbf{C}_{n \times n}$ and $\xi=1$ or -1 such that

$$
T(A)=\xi U A U^{*} \text { for all } A \text { in } \mathcal{H}_{n},
$$

or

$$
T(A)=\xi(A)=\xi U A^{T} U^{*} \quad \text { for all } A \text { in } \sharp_{n} .
$$

Received by the editors April 17, 1985 and, in revised form, February 27, 1986.

1980 Mathematics Subject Classification (1985 Revision). Primary 15A04.

The author wishes to express his thanks to Dr. Yik-Hoi Au-Yeung for his valuable advice and encouragement. 
As shown in the next section, by Theorems 1 and 2 we can deduce

Theorem 3. Let T: $\mathbf{C}_{n \times n} \rightarrow \mathbf{C}_{n \times n}$ be a linear transformation. The following are equivalent.

(a) $W(T(A))=W(A)$ for all $A$ in $\mathbf{C}_{n \times n}$.

(b) $W(T(A))=W(A)$ for all $A$ in $\nvdash_{n}$.

(c) There exists a unitary matrix $U$ in $\mathbf{C}_{n \times n}$ such that

$$
T(A)=U A U^{*} \text { for all } A \text { in } \mathbf{C}_{n \times n},
$$

or

$$
T(A)=U A^{T} U^{*} \quad \text { for all } A \text { in } \mathbf{C}_{n \times n} .
$$

We note that the equivalence of conditions (a) and (c) in Theorem 3 is the matrix version of a result of Pellegrini $[\mathbf{5}]$ concerning the numerical range preserving operators on a Banach algebra.

Since the numerical range of a Hermitian matrix is just the convex hull of its eigenvalues (see [1, Corollary 4.7]), by the equivalence of conditions (b) and (c) in Theorem 3 we have

Corollary $1[\mathbf{3}]$. A linear operator $T: \mathbf{C}_{n \times n} \rightarrow \mathbf{C}_{n \times n}$ satisfies (i) $T\left(\mathscr{H}_{n}\right) \subset$ $H_{n}$, and (ii) $T(A)$ and $A$ have the same eigenvalues for all $A \in H_{n}$, if and only if condition (c) of Theorem 3 holds.

Let $\mathcal{G}_{n}$ be the real linear space of all $n \times n$ skew-Hermitian matrices. For any linear operator $T: \mathcal{G}_{n} \rightarrow \mathcal{G}_{n}$, we have a corresponding linear operator $\hat{T}: \mathfrak{k}_{n} \rightarrow \mathfrak{H}_{n}$ defined by

$$
\hat{T}(A)=i T(i A) \quad \text { for all } A \text { in } \varkappa_{n} .
$$

Consequently by Theorem 2 we have

COROLlaRY 2. A linear operator $T: \mathcal{G}_{n} \rightarrow \mathcal{G}_{n}$ satisfies

$$
r(T(A))=r(A) \text { for all } A \text { in } \mathcal{G}_{n}
$$

if and only if there exist a unitary matrix $U$ in $\mathbf{C}_{n \times n}$ and $\xi=1$ or -1 such that

$$
T(A)=\xi U A U^{*} \text { for all } A \text { in } \mathcal{G}_{n},
$$

or

$$
T(A)=\xi U A^{T} U^{*} \quad \text { for all } A \text { in } \mathcal{G}_{n} .
$$

Proofs. In the following let $\mathcal{U}_{n}$ be the group of unitary matrices in $\mathbf{C}_{n \times n}$ and denote by $E_{j k}$ the matrix in $\mathbf{C}_{n \times n}$ with the $j-k$ entry, which equals 1 , as the only nonzero entry. We note that a complex number $z$ is in $W(A)$ if and only if there exists $U$ in $U_{n}$ such that the 1-1 entry of $U A U^{*}$ equals $z$. Moreover, the following properties of the numerical radius will be used frequently in the proofs.

(a) For any $A$ in $\mathbf{C}_{n \times n}, U$ in $\mathcal{U}_{n}, r(A)=r\left(A^{T}\right)=r\left(U A U^{*}\right)$.

(b) If $A_{1}$ is a principal submatrix of $A$, then $r\left(A_{1}\right) \leq r(A)$.

(c) If $A \in \mathscr{H}_{n}$, then $r(A)=\rho(A)$; here $\rho(\cdot)$ denotes the spectral radius.

We begin with the following lemmas. 
LEMMA 1. If

$$
A=\left(\begin{array}{cc}
1 & a_{12} \\
a_{21} & a_{22}
\end{array}\right)
$$

in $\mathbf{C}_{n \times n}$ is such that $r(A)=1$, then $a_{12}=-\overline{a_{21}}$.

Proof. Suppose that $A$ satisfies the hypothesis of the lemma. Let $H=$ $\left(A+A^{*}\right) / 2$. Then 1 is the $1-1$ entry of $H$ and hence $1 \leq r(H) \leq r(A) \leq 1$. Since $r(H)=\rho(H), 1$ must be the largest eigenvalue of $H$. Thus the 1-2 and 2-1 entries of $H$ are equal to zero, i.e., $a_{12}=-\overline{a_{21}}$.

LEMMA 2. Let $A=\left(a_{i j}\right), B=\left(b_{i j}\right) \in \mathbf{C}_{2 \times 2}$ be such that $a_{11}=1=r(A)$. If $r(A+\theta B) \leq 1$ for all $\theta$ in $\mathbf{C}$ with $|\theta|=1$, then $b_{11}=b_{12}=b_{21}=0$.

Proof. Let $D(\theta)=A+\theta B$. Since the 1-1 entry of $D(\theta)$ is $a+\theta b_{11}$, if $r(D(\theta)) \leq 1$ for all $\theta$ in $\mathbf{C}$ with $|\theta|=1$, then $\left|1+\theta b_{11}\right| \leq 1$ for all $\theta$ in $\mathbf{C}$ with $|\theta|=1$. Thus $b_{11}=0$. Moreover, if $b_{12}$ and $b_{21}$ are not both zero, we can always find $\theta$ in $\mathbf{C}$ with $|\theta|=1$ such that $a_{12}+\theta b_{12} \neq-\left(\overline{a_{21}+\theta b_{21}}\right)$. By Lemma 1 , we have $r(D(\theta))>1$, which contradicts our assumption. Therefore $b_{12}=b_{21}=0$.

LEMMA 3. Let $A \in \mathbf{C}_{n \times n}$. If $r(\theta A+I) \leq 1$ for all $\theta$ in $\mathbf{C}$ with $|\theta|=1$, then $A=0$.

Proof. Let $A \in \mathbf{C}_{n \times n}$ satisfy the hypothesis of the lemma. Suppose $x$ is a unit vector in $\mathbf{C}^{n}$ such that $x A x *=\theta r(A)$, where $\theta \in \mathbf{C}$ with $|\theta|=1$. If $A \neq 0$, then $r(A)>0$ and hence

$$
\begin{aligned}
1<r(A)+1 & =|\theta r(A)+\theta|=\left|x A x^{*}+\theta\right| \\
& =\left|x(A+\theta I) x^{*}\right| \leq r(A+\theta I) \leq 1,
\end{aligned}
$$

which is a contradiction. Thus $A=0$.

LEMMA 4. A matrix $U$ in $\mathbf{C}_{n \times n}$ satisfies

$$
r(U A)=r(A) \text { for all } A \text { in } \mathbf{C}_{n \times n}
$$

if and only if $U=\xi I$ for some $\xi$ in $\mathbf{C}$ with $|\xi|=1$.

Proof. $(\Leftarrow)$ Clear.

$(\Rightarrow)$ Let $U \in \mathbf{C}_{n \times n}$ with eigenvalues $u_{1}, \ldots, u_{n}$ satisfy the hypothesis of the lemma. Then for $k=1, \ldots, n$, we can find $W_{k} \in U_{n}$ such that $W_{k} U W_{k}^{*}$ is in upper triangular form with $u_{k}$ as the 1-1 entry. Let $A_{k}=W_{k}^{*} E_{11} W_{k}$. Then

$$
\begin{aligned}
1 & =r\left(A_{k}\right)=r\left(U W_{k}^{*} E_{11} W_{k}\right)=r\left(W_{k} U W_{k}^{*} E_{11}\right) \\
& =r\left(u_{k} E_{11}\right)=\left|u_{k}\right|,
\end{aligned}
$$

$k=1, \ldots, n$. Let $W \in \mathcal{U}_{n}$ be such that

$$
V=W U W^{*}=\left(\begin{array}{ccc}
u_{1} & & 0 \\
& \ddots & \\
\left(u_{i j}\right) & & u_{n}
\end{array}\right) .
$$

We have $r(V A)=r(A)$ for all $A$ in $\mathbf{C}_{n \times n}$. In particular, for $k=1, \ldots, n-1$, $r\left(E_{k k}\right)=r\left(V E_{k k}\right)$. If there exists $u_{j k} \neq 0(j>k)$, then by the elliptical range 
theorem (see [4]), $u_{k}$ is one of the foci of the nondegenerated elliptical disk $W(B)$, where

$$
B=\left(\begin{array}{cc}
u_{k} & 0 \\
u_{j k} & 0
\end{array}\right)
$$

is the submatrix of $V E_{k k}$. Hence

$$
r\left(E_{k k}\right)=1=\left|u_{k}\right|<r(B) \leq r\left(V E_{11}\right),
$$

which is a contradiction. Thus $V$ is in diagonal form. Let $u_{1}=\xi$. Suppose there exists $u_{j} \neq \xi$, where $1<j \leq n$. Consider $A=E_{11}+E_{1 j}-E_{j 1}-E_{j j} \in \mathbf{C}_{n \times n}$. Then $r(A)=1$. Consider the submatrix

$$
B=\left(\begin{array}{cc}
\xi & \xi \\
-u_{j} & -u_{j}
\end{array}\right)
$$

of the matrix $V A$. As $\bar{\xi} u_{j} \neq 1$, by Lemma 1

$$
1<r(\bar{\xi} B)=r(B) \leq r(V A)=r(A)=1,
$$

which is a contradiction. Thus $\xi=u_{j}, j=1, \ldots, n$. Hence $U=W^{*} V W=\xi I$.

PROOF OF THEOREM 1. $(\Leftarrow)$ Clear.

$(\Rightarrow)$ We prove the statement by induction on $n$. For $n=1$ the statement is obvious. Let $n \geq 2$ and $T: \mathbf{C}_{n \times n} \rightarrow \mathbf{C}_{n \times n}$ satisfy the hypothesis of the statement. We first show that $T\left(\mathcal{U}_{n}\right) \subset \mathcal{U}_{n}$. Let

$$
U=X\left(\sum_{j=1}^{n} \xi_{j} E_{j j}\right) X * \in \mathcal{U}_{n}
$$

where $X \in \mathcal{U}_{n}$ and $\left|\xi_{j}\right|=1, j=1, \ldots, n$. If $A=\xi_{1} X E_{11} X^{*}$, then $1=r(A)=$ $r(T(A))$. Hence there exists $Y$ in $U_{n}$ such that $T(A)=Y A^{\prime} Y^{*}$, where the 1-1 entry of $A^{\prime}$ is $\eta$ with $|\eta|=1$. Instead of $T$ we consider $T^{\prime}$ defined by

$$
T^{\prime}(B)=\bar{\eta} Y^{*} T\left(\xi_{1} X B X^{*}\right) Y \quad \text { for all } B \text { in } \mathbf{C}_{n \times n} .
$$

Then $T^{\prime}$ is also numerical radius preserving and

$$
T^{\prime}\left(E_{11}\right)=\left(a_{j k}\right) \quad \text { with } a_{11}=1 .
$$

Let $S=\left\{E_{j k}: 2 \leq j, k \leq n\right\}$ and denote by $\langle S\rangle$ the linear subspace in $\mathbf{C}_{n \times n}$ spanned by $S$. Suppose $E \in S$ and $T^{\prime}(E)=\left(b_{j k}\right)$. Note that $r\left(E_{11}+\theta E\right)=1$ for all $\theta$ in $\mathbf{C}$ with $|\theta|=1$. Let

$$
B_{j}=\left(\begin{array}{ll}
b_{11} & b_{1^{\prime} j} \\
b_{j 1} & b_{j j}
\end{array}\right), \quad A_{j}=\left(\begin{array}{cc}
1 & a_{1 j} \\
a_{j 1} & a_{j j}
\end{array}\right) \quad(2 \leq j \leq n),
$$

be the submatrices of $T^{\prime}(E)$ and $T^{\prime}\left(E_{11}\right)$, respectively. Then we have

$$
r\left(A_{j}+\theta B_{j}\right) \leq 1 \text { for all } \theta \text { in } \mathbf{C} \text { with }|\theta|=1 .
$$

By Lemma $2, b_{1 j}=b_{j 1}=b_{11}=0(2 \leq j \leq n)$. Thus $T^{\prime}(E) \in\langle S\rangle$ and hence $T^{\prime}(\langle S\rangle) \subset\langle S\rangle$. Clearly, the restriction of $T^{\prime}$ on $\langle S\rangle$ is a numerical radius preserving linear operator. By the induction assumption, there exist $W$ in $U_{n-1}$ and $\delta$ in $\mathbf{C}$ with $|\delta|=1$ such that

(i) $T^{\prime}((0) \oplus A)=(0) \oplus \delta W A W^{*}$ for all $A$ in $\mathbf{C}_{(n-1) \times(n-1)}$, or 
(ii) $T^{\prime}((0) \oplus A)=(0) \oplus \delta W A^{T} W^{*}$ for all $A$ in $\mathbf{C}_{(n-1) \times(n-1)}$. In both cases we have $T^{\prime}\left(\bar{\delta} \sum_{j=2}^{n} E_{j j}\right)=\sum_{j=2}^{n} E_{j j}$. Now consider

$$
D(\theta)=T^{\prime}\left(\theta E_{11}+\bar{\delta} \sum_{j=2}^{n} E_{j j}\right)=\theta\left(a_{j k}\right)+\sum_{j=2}^{n} E_{j j}
$$

We have $r(D(\theta))=r\left(\theta E_{11}+\bar{\delta} \sum_{j=2}^{n} E_{j j}\right)=1$ for all $\theta$ in $\mathbf{C}$ with $|\theta|=1$. Hence the submatrix $A^{\prime}=\left(a_{j k}\right)_{2 \leq j, k \leq n}$ of $T^{\prime}\left(E_{11}\right)$ satisfies

$$
r\left(\theta A^{\prime}+I\right) \leq 1 \text { for all } \theta \text { in } \mathbf{C} \text { with }|\theta|=1 .
$$

By Lemma 3 we have $A^{\prime}=0$. Moreover, for any submatrix

$$
C(\theta)=\theta\left(\begin{array}{cc}
a_{11} & a_{1 j} \\
a_{j 1} & 0
\end{array}\right)+\left(\begin{array}{cc}
0 & 0 \\
0 & 1
\end{array}\right)
$$

where $2 \leq j \leq n$, of $D(\theta)$ we have

$$
1 \geq r(C(\theta)) \text { for all } \theta \text { in } \mathbf{C} \text { with }|\theta|=1 .
$$

By Lemma $2 a_{1 j}=a_{j 1}=0$ and hence $\left(a_{j k}\right)=E_{11}$. Consequently,

$$
T^{\prime}\left(\operatorname{diag}\left(1, \bar{\xi}_{1} \xi_{2}, \ldots, \bar{\xi}_{1} \xi_{n}\right)\right)
$$

is unitary and hence $T(U)$ is unitary. Therefore $T\left(U_{n}\right) \subset U_{n}$ and by the result of Marcus [2], there exist $P, Q$ in $U_{n}$ such that

(i) $T(A)=P A Q$ for all $A$ in $\mathbf{C}_{n \times n}$, or

(ii) $T(A)=P A^{T} Q$ for all $A$ in $\mathbf{C}_{n \times n}$.

If (i) holds, then for all $A$ in $\mathbf{C}_{n \times n}$,

$$
r(A)=r(T(A))=r(P A Q)=r\left(Q P A Q Q^{*}\right)=r(Q P A)
$$

implies $Q P=\xi I$ with $|\xi|=1$ by Lemma 4 . Therefore $T(A)=\xi P A P^{*}$ for all $A \in \mathbf{C}_{n \times n}$.

If (ii) holds, then for all $A$ in $\mathbf{C}_{n \times n}$,

$$
r(A)=r(T(A))=r\left(P A^{T} Q\right)=r\left(P^{*} P A^{T} Q P\right)=r\left(A^{T} Q P\right)=r\left((Q P)^{T} A\right)
$$

implies $(Q P)^{T}=\xi I$ with $|\xi|=1$ by Lemma 4. Therefore $T(A)=\xi P A^{T} P^{*}$ for all $A \in \mathbf{C}_{n \times n}$.

To prove Theorem 2 we need the following lemmas.

LEMMA 5. Let $A=\left(a_{j k}\right) \in \mathscr{H}_{n}$. If $\left|a_{l l}\right|=r(A)$, then

$$
a_{l k}=a_{k l}=0 \text { for all } k=1, \ldots, l-1, l+1, \ldots, n \text {. }
$$

Proof. Let $A$ satisfy the hypothesis of the lemma. If $\left|a_{l l}\right|=r(A)=\rho(A)$, then $a_{l l}$ is the largest or the smallest eigenvalue of $H$. The result follows.

LEMMA 6. Let $A \in \mathbf{C}_{n \times n}$. Then $r(A) \leq\left(\operatorname{tr} A A^{*}\right)^{1 / 2}$. The equality holds if and only if $A$ is a normal matrix of rank 1 .

Proof. Let $A \in \mathbf{C}_{n \times n}$ have singular values $a_{1} \geq \cdots \geq a_{n}$. Then

$$
r(A) \leq a_{1} \leq\left(\sum_{j=1}^{n} a_{j}^{2}\right)^{1 / 2}=\left(\operatorname{tr} A A^{*}\right)^{1 / 2}
$$

with equality if and only if $a_{2}=\cdots=a_{n}=0$, and $a_{1}=r(A)$, i.e., $A$ is unitarily similar to $z E_{11}$, where $z \in \mathbf{C}$ with $|z|=a_{1}$ (see [1, Theorem 5.5]). 
LEMMA 7. Let $T: \mathscr{H}_{2} \rightarrow \mathscr{H}_{2}$ be a linear operator such that $T\left(E_{11}\right)=E_{11}$ and

$$
r(T(A))=r(A) \text { for all } A \text { in } \mathfrak{H}_{2} \text {. }
$$

Suppose that $S=\left\{E_{12}+E_{21}, i E_{12}-i E_{21}\right\} \subset \mathcal{H}_{2}$ and $\langle S\rangle$ denotes the linear subspace of $\mathcal{H}_{2}$ spanned by $S$. Then $T\left(E_{22}\right)=E_{22}$ and $T(\langle S\rangle) \subset\langle S\rangle$. Consequently $T\left(E_{12}+E_{21}\right)=\lambda E_{12}+\bar{\lambda} E_{21}$ and $T\left(i E_{12}-i E_{21}\right)=\mu i E_{12}-\bar{\mu} i E_{21}$ for some $\lambda, \mu$ in $\mathbf{C}$ with $|\lambda|=|\mu|=1$.

Proof. Suppose that the hypotheses of the lemma hold and let

$$
T\left(E_{22}\right)=\left(\begin{array}{ll}
a_{11} & a_{12} \\
\overline{a_{12}} & a_{22}
\end{array}\right) \in \mathscr{H}_{2} .
$$

Then the 1-1 entry of $T\left(E_{11} \pm E_{22}\right)$ is $1 \pm a_{11}$. Since

$$
1=r\left(E_{11} \pm E_{22}\right)=r\left(T\left(E_{11} \pm E_{22}\right)\right),
$$

we have $\left|1+a_{11}\right| \leq 1$ and hence $a_{11}=0$. Now the 1-1 entry of $T\left(E_{11}+E_{22}\right)$ equals $1=r\left(T\left(E_{11}+E_{22}\right)\right)$, and by Lemma $5, a_{12}=0$. Consequently $T\left(E_{22}\right)=a_{22} E_{22}$. As $1=r\left(E_{22}\right)=r\left(T\left(E_{22}\right)\right)$, we have $a_{22}=1$ or -1 .

Now consider $B=\alpha E_{12}+\bar{\alpha} E_{21}$, where $|\alpha|=1$. Let $T(B)=\left(b_{i j}\right) \in \mathfrak{H}_{2}$. If $b_{11} \notin 0$, we can find $t>0$ such that $\left|2 t b_{11}\right|>1$. Then the 1-1 entry of $T\left(2 t E_{11} \pm B\right)$ will be $2 t \pm b_{11}$ and hence we have

$$
r\left(T\left(2 t E_{11}+B\right)\right) \geq 2 t+b_{11}>t+\sqrt{t^{2}+1}=r\left(2 t E_{11}+B\right),
$$

or

$$
r\left(T\left(2 t E_{11}-B\right)\right) \geq 2 t-b_{11}>t+\sqrt{t^{2}+1}=r\left(2 t E_{11}-B\right),
$$

which is a contradiction. Thus $b_{11}=0$. As $T\left(E_{22}\right)=a_{22} E_{22}$, where $a_{22}=1$ or -1 , we can prove $b_{22}=0$ by similar arguments. As a result, $T(B)=b_{12} E_{12}+\overline{b_{12}} E_{21}$. Putting $\alpha=1$ and $i$, we get the last statement of the lemma.

Now consider $a_{22}$. Let $C=E_{11}+E_{12}+E_{21}+E_{22} \in \mathcal{H}_{2}$. Then $T(C)=$ $E_{11}+\lambda E_{12}+\bar{\lambda} E_{21}+a_{22} E_{22}$. Since $C$ is of rank 1 , by Lemma 6

$$
2=\left(\operatorname{tr} C^{*}\right)^{1 / 2}=r(C)=r(T(C)) \leq\left(\operatorname{tr} T(C) T(C)^{*}\right)^{1 / 2}=2 .
$$

Therefore $T(C)$ is of rank 1 and hence $a_{22}=1$.

PROOF OF THEOREM 2. $(\Leftarrow)$ Clear.

$\Rightarrow$ We prove the statement by induction on $n$. For $n=1$ the statement is obvious. Let $n \geq 2$ and $T: \mathfrak{H}_{n} \rightarrow \mathfrak{H}_{n}$ satisfy the hypothesis of the statement. Suppose

$$
T\left(E_{11}\right)=U\left(\sum_{j=1}^{n} a_{j} E_{j j}\right) U^{*} \quad \text { where } a_{1}, \ldots, a_{n} \in \mathbf{R}
$$

and $\left|a_{n}\right| \leq\left|a_{n-1}\right| \leq \cdots \leq\left|a_{1}\right|=r\left(T\left(E_{11}\right)\right)=1$. Instead of $T$, we consider $T^{\prime}$ defined by

$$
T^{\prime}(A)=a_{11} U^{*}(T(A)) U
$$

Then $T^{\prime}$ is also numerical radius preserving and

$$
T^{\prime}\left(E_{11}\right)=E_{11}+\sum_{j=2}^{n} b_{j} E_{j j} \quad \text { with } 1 \geq\left|b_{2}\right| \geq \cdots \geq\left|b_{n}\right| .
$$


Let $S=\left\{E \in \mathbf{C}_{n \times n}: E=E_{j j}\right.$ or $E_{j k}+E_{k j}$ or $\left.i E_{j k}-i E_{k j}, 2 \leq j, k \leq n\right\}$ and denote by $\langle S\rangle$ the linear subspace in $\mathbf{C}_{n \times n}$ spanned by $S$. Suppose $E \in S$ and $T^{\prime}(E)=\left(c_{j k}\right)$. Then $c_{11}=0$, otherwise the 1-1 entry of $T^{\prime}\left(E_{11}+E\right)$ or $T^{\prime}\left(E_{11}-E\right)$ will be greater than $1=r\left(E_{11} \pm E\right)=r\left(T\left(E_{11} \pm E\right)\right)$. Moreover, since the 1-1 entry of $T\left(E_{11}+E\right)$ equals $r\left(T\left(E_{11}+E\right)\right)$, by Lemma $5, c_{1 j}=c_{j 1}=0$ for $2 \leq j \leq n$. Thus $T^{\prime}(E) \in\langle S\rangle$ and hence $T^{\prime}(\langle S\rangle) \subset\langle S\rangle$. Clearly, the restriction of $T^{\prime}$ on $\langle S\rangle$ is a numerical radius preserving linear operator. By the induction assumption, there exixt $V$ in $U_{n-1}$ and $\delta=1$ or -1 such that

(i) $T^{\prime}((0) \oplus A)=(0) \oplus \delta V A V^{*}$ for all $A$ in $\nvdash_{n-1}$, or

(ii) $T^{\prime}((0) \oplus A)=(0) \oplus \delta V A^{T} V^{*}$ for all $A$ in $\nvdash_{n-1}$.

Now $T^{\prime}\left(E_{11} \pm \delta \sum_{j=2}^{n} E_{j j}\right)=E_{11}+\sum_{j=2}^{n}\left(b_{j} \pm 1\right) E_{j j}$. As $r\left(T^{\prime}\left(E_{11} \pm \delta \sum_{j=2}^{n} E_{j j}\right)\right)=$ $1 \geq\left|b_{j} \pm 1\right|$, we have $b_{j}=0, j=2, \ldots, n$, and hence $T^{\prime}\left(E_{11}\right)=E_{11}$.

If $n=2$, then $T^{\prime}$ satisfies the hypothesis of Lemma 7. Thus $T^{\prime}\left(E_{22}\right)=E_{22}$, $T^{\prime}\left(E_{12}+E_{21}\right)=\lambda E_{12}+\bar{\lambda} E_{21}$ and $T^{\prime}\left(i E_{12}-i E_{21}\right)=\mu i E_{12}=\bar{\mu} i E_{21}$, where $|\lambda|=|\mu|=1$. Let $B=(1+i) E_{12}+(1-i) E_{21}$. Then

$$
\sqrt{2}=r(B)=r\left(T^{\prime}(B)\right)=|\lambda+\mu i|
$$

implies $\lambda=\mu$ or $\lambda=-\mu$. Thus

$$
T^{\prime}(A)= \begin{cases}D A D^{*} & \text { if } \lambda=\mu, \\ D A^{T} D^{*} & \text { if } \lambda=-\mu,\end{cases}
$$

where $D=\lambda E_{11}+E_{22}$. Hence $T$ is of the required form.

Suppose $n \geq 3$. Instead of $T^{\prime}$, we consider $T^{0}$ defined by

$$
T^{0}(A)= \begin{cases}V_{1} T^{\prime}(A) V_{1}^{*} & \text { if (i) is true, } \\ {\left[V_{1} T^{\prime}(A) V_{1}^{*}\right]^{T}} & \text { if (ii) is true }\end{cases}
$$

where $V_{1}=(1) \oplus V^{*}$. Then $T^{0}$ is numerical radius preserving and

$$
T^{0}\left(E_{11}\right)=E_{11}, \quad T^{0}((0) \oplus A)=(0) \oplus \delta A \quad \text { for all } A \in \nvdash_{n-1} .
$$

Now if $B=\alpha E_{1 j}+\bar{\alpha} E_{j 1}$, where $2 \leq j \leq n,|\alpha|=1$, and $T^{0}(B)=\left(b_{l k}\right)$, then the $k$ - $k$ entry of $T^{0}\left(\sum_{l \neq 1, j} E_{l l} \pm B\right)$ is $\delta \pm b_{k k}$ for $k \neq 1, j$. Thus $1=r\left(\sum_{l \neq 1, j} E_{l l} \pm B\right)=$ $r\left(T^{0}\left(\sum_{l \neq 1, j} E_{l l} \pm B\right)\right) \geq\left|\delta \pm b_{k k}\right|$ implies $b_{k k}=0$ for $k \neq 1, j$. Moreover, since the $k$ - $k$ entry of $T^{0}\left(\sum_{l \neq 1, j} E_{l l}+B\right)$ equals $\delta$ for $k \neq 1, j$, by Lemma $6, b_{l k}=0$ except $(l, k)=(1,1),(1, j),(j, 1)$, and $(j, j)$. Hence, if $S_{j}=\left\{E_{11}, E_{j j}, E_{1 j}+E_{j 1}, i E_{1 j}-\right.$ $\left.i E_{j 1}\right\}$, then $T^{0}\left(\left\langle S_{j}\right\rangle\right) \subset\left\langle S_{j}\right\rangle$. Note that the restriction of $T^{0}$ on $\left\langle S_{j}\right\rangle$ satisfies the hypothesis of Lemma 7. As a result, we have $\delta=1, T^{0}\left(E_{1 j}+E_{j 1}\right)=\lambda_{j} E_{1 j}+\bar{\lambda}_{j} E_{j 1}$ and $T^{0}\left(i E_{1 j}-i E_{j 1}\right)=\mu_{j} i E_{1 j}-\bar{\mu}_{j} i E_{j 1}$, where $\left|\lambda_{j}\right|=\left|\mu_{j}\right|=1, j=2, \ldots, n$. We may assume $\lambda_{2}=1$; otherwise consider $T^{\prime \prime}$ defined by

$$
T^{\prime \prime}(A)=W\left(T^{0}(A)\right) W^{*} \text { for all } A \text { in } \nvdash_{n},
$$

where $W=\bar{\lambda}_{2} E_{11}+\sum_{j=2}^{n} E_{j j}$, instead of $T^{0}$. Then let $B=\sum_{1 \leq j, k \leq n} E_{j k}$. We have

$$
T^{0}(B)=\sum_{2 \leq j, k \leq n} E_{j k}+E_{11}+E_{12}+E_{21}+\sum_{j=3}^{n}\left(\lambda_{j} E_{1 j}+\bar{\lambda}_{j} E_{j 1}\right) .
$$

Since $B$ is of rank 1 , by Lemma 6 ,

$$
n=\left(\operatorname{tr} B B^{*}\right)^{1 / 2}=r(B)=r\left(T^{0}(B)\right) \leq\left(\operatorname{tr} T^{0}(B) T^{0}(B)^{*}\right)^{1 / 2}=n .
$$


Thus $T^{0}(B)$ is also of rank 1 and hence $\lambda_{j}=1(j=3, \ldots, n)$. For $\mu_{2}$ we consider

$$
B=\sum_{l=1}^{3} E_{l l}+E_{13}+E_{31}-i E_{23}+i E_{32}+i E_{12}-i E_{21} \text {. }
$$

We have

$$
T^{0}(B)=\sum_{l=1}^{3} E_{l l}+E_{13}+E_{31}-i E_{23}+i E_{32}+\mu_{2} i E_{12}-\bar{\mu}_{2} i E_{21} .
$$

Since $B$ is of rank 1 , by Lemma 6 ,

$$
3=\left(\operatorname{tr} B B^{*}\right)^{1 / 2}=r(B)=r\left(T^{0}(B)\right) \leq\left(\operatorname{tr} T^{0}(B) T^{0}(B)^{*}\right)^{1 / 2}=3 .
$$

Thus $T^{0}(B)$ is also of rank 1 and hence $\mu_{2}=1$. For $\mu_{j}$ with $j \geq 3$, we consider

$$
B=E_{11}+E_{22}+E_{j j}+E_{2 j}+E_{j 2}+i E_{12}-i E_{21}+i E_{1 j}-i E_{j 1} \text {. }
$$

Then $B$ is of rank 1 and by the same argument as above, $\mu_{j}=1$ for $3 \leq j \leq n$. Consequently $T^{0}(A)=A$ for all $A$ in $\mathcal{H}_{n}$ and hence $T$ is of the required form.

ProOF OF THEOREM 3. (c) $\Rightarrow(\mathrm{a}) \Rightarrow(\mathrm{b})$. Clear.

(b) $\Rightarrow$ (c). Note that $A \in \mathscr{H}_{n}$ if and only if $W(A) \subset \mathbf{R}$. If

$$
W(T(A))=W(A) \text { for all } A \in \mathscr{H}_{n},
$$

then $T\left(\mathscr{H}_{n}\right) \subset \mathfrak{H}_{n}$. Thus the restriction of $T$ on $\mathfrak{H}_{n}$ may be regarded as a real linear operator such that

$$
r(T(A))=r(A) \text { for all } A \in \mathcal{H}_{n} .
$$

By Theorem 3, there exist $U$ in $U_{n}$ and $\xi=1$ or -1 such that (1.3) or (1.4) holds. Since $W(T(I))=W(I)$, we have $\xi=1$. Finally, for any $A \in \mathbf{C}_{n \times n}$,

$$
T(A)=T(H)+i T(G)=U H U^{*}+i U G U^{*}=U A U^{*},
$$

where $H=\left(A+A^{*}\right) / 2, G=i\left(A^{*}-A\right) / 2$. Hence $U$ is the unitary matrix such that (1.5) or (1.6) holds.

\section{REFERENCES}

1. C. K. Li, T. Y. Tam and N. K. Tsing, The generalized spectral radius, numerical radius and spectal norm, Linear and Multilinear Algebra 16 (1984), 215-237.

2. M. Marcus, All linear operators leaving the unitary group invariant, Duke Math. J. 26 (1959), 155-163.

3. M. Marcus and B. N. Moyls, Linear transformation on algebras of matrices, Canad. J. Math. 11 (1959), 61-66.

4. F. D. Murnaghan, On the field of values of a square matrix, Proc. Nat. Acad. Sci. U.S.A. 18 (1932), 246-248.

5. V. J. Pellegrini, Numerical range preserving operators on a Banach algebra, Studia Math. 54 (1975), 143-147.

Department of Mathematics, University of Hong Kong, Hong Kong

Current address: Department of Mathematics, University of Wisconsin, Madison, Wisconsin 53706 